Postprint version of the manuscript published in The Astrophysical Journal 909 (2021): 175.

\title{
Effects of forcing mechanisms on the multiscale properties of magnetohydrodynamics
}

\author{
Yan Yang,${ }^{1}$ Moritz Linkmann, ${ }^{2}$ Luca Biferale,${ }^{3}$ And Minping Wan ${ }^{1,4}$ \\ ${ }^{1}$ Department of Mechanics and Aerospace Engineering, Southern University of Science and Technology, Shenzhen 518055, China \\ ${ }^{2}$ School of Mathematics and Maxwell Institute for Mathematical Sciences, University of Edinburgh, Edinburgh EH9 $3 F D$, United Kingdom \\ ${ }^{3}$ Department of Physics and INFN, University of Rome "Tor Vergata", Via della Ricerca Scientifica 1, I-00133 Rome, Italy \\ ${ }^{4}$ Guangdong Provincial Key Laboratory of Turbulence Research and Applications, Southern University of Science and Technology, \\ Shenzhen, Guangdong 518055, China
}

\begin{abstract}
We performed numerical simulations to study the response of magnetohydrodynamics (MHD) to large-scale stochastic forcing mechanisms parametrized by one parameter, $0 \leq a \leq 1$, going from direct injection on the velocity field $(a=1)$ to stirring acts on the magnetic field only $(a=0)$. We study the multi-scale properties of the energy transfer, by splitting the total flux in channels mediated by (i) the kinetic non-linear advection, (ii) the Lorentz force, (iii) the magnetic advection and (iv) magnetic stretching term. We further decompose the fluxes in two sub-channels given by heterochiral and homochiral components in order to distinguish forward, inverse and flux-loop cascades. We show that there exists a quasi-singular role of the magnetic forcing mechanism for $a \sim 1$ : a small injection on the magnetic field $a<1$ can strongly deplete the mean flux of kinetic energy transfer throughout the kinetic non-linear advection channel. We also show that this negligible mean flux is the result of a flux-loop balance between heterochiral (direct) and homochiral (inverse) transfers. Conversely, both homochiral and heterochiral channels transfer energy forward for the other three channels. Cross exchange between velocity and the magnetic field is reversed around $a=0.4$ and except when $a \sim 1$ we always observe that heterochiral mixed velocity-magnetic energy triads tend to move energy from magnetic to velocity fields. Our study is an attempt to further characterize the multi-scale nature of MHD dynamics, by disentangling different properties of the total energy transfer mechanisms, which can be useful for improving sub-grid-modelling.
\end{abstract}

\section{INTRODUCTION}

The turbulent dynamics of conducting fluids such as liquid metals and plasmas in the one-fluid approximation, are relevant to a variety of observed phenomena in astro- and geophysical fluid dynamics. The most pertinent examples are stellar and planetary dynamos (Moffatt 1978; Parker 1979; Brandenburg \& Subramanian 2005; Tobias et al. 2013), the granulation in the solar convection zone (Leighton et al. 1962; Leighton 1963; Bray et al. 1984; Rieutord \& Rincon 2010), and turbulence in the solar wind and Earth's magnetosheath (Barnes 1979; Tu \& Marsch 1995; Goldstein et al. 1995; Bruno \& Carbone 2005). All these systems can be described to a good approximation by the equations of magnetohydrodynamics (MHD), albeit at different parameter ranges, and potentially coupled to additional evolution equations. The magnetic activity of the Sun, for instance, originates from the solar tachocline (Mi-

wanmp@sustech.edu.cn esch 2005) and the main features of its dynamics can be well described by an ideal, i.e. dissipationless, MHD shallow-water model (Gilman 2000; Dikpati \& Gilman 2001). The latter highlights an important unresolved challenge in astrophysical fluid dynamics - both magnetic and fluid Reynolds numbers are usually very high, leading to turbulent dynamics on scales much too small to be adequately resolved in numerical simulations. The parameter ranges are way beyond the capabilites even for optimised codes running on state-of-the-art highperformance computing facilities. Observational studies give very important insights (Tu \& Marsch 1995; Horbury et al. 2005; Chen 2016; Bruno \& Carbone 2013; Matthaeus et al. 2019; Klein et al. 2019), however the data acquisition process is complex and high-order statistics are difficult to measure precisely.

To further disentangle the complexity of MHD turbulence, a better understanding of the intricate multiscale transfer of inertial conserved quantities as the total energy, the magnetic and the cross helicity is mandatory (Mininni 2011; Biskamp 2003; Galtier 2016; Zhou et al. 2004; Alexakis \& Biferale 2018). The overall global 
picture might strongly depend on the injection properties, being the three conserved quantities dynamically correlated. As a result, systematic studies at varying one or a few control parameters, connected to the properties of the stirring mechanisms are important for both fundamental and applied interests, e.g. concerning the issue of universality - independence of the forcing and small-scale modelling (Alexakis 2013; Bian \& Aluie 2019; McKay et al. 2017; Zhou \& Vahala 1991; Müller \& Carati 2002; Chernyshov et al. 2010; Kessar et al. 2016; Miesch et al. 2015).

In recent years, it has become more and more clear that global measurements based on spectra and mean fluxes do not allow for a precise disentanglement of the physics mechanisms underlying the multi-scale transport, e.g. direct or inverse energy cascades can have the same spectral properties. In MHD turbulence the situation is particularly complicated, as the interaction of magnetic and velocity fluctuations can proceed in different parameter regimes. A background magnetic field or different levels of cross- and magnetic helicities, for instance, have measureable effects on the value of the scaling exponent of magnetic and kinetic energy spectra. The complexity of the problem is reflected in the considerable effort that has been put upon measuring and understanding spectral scaling and other secondorder statistics for the incompressible case (Iroshnikov 1964; Kraichnan 1965; Matthaeus \& Zhou 1989; Goldreich \& Sridhar 1995; Müller \& Biskamp 2000; Galtier et al. 2000, 2002; Boldyrev 2005a,b; Mason et al. 2006; Boldyrev et al. 2009; Beresnyak \& Lazarian 2009; Grappin \& Müller 2010). More recently, effects specific to compressible flows have been quantified (Teissier \& Müller 2020; Grete et al. 2020).

Similarly, even the sign of the total flux does not fully summarise the entire flow dynamics, we know examples where the mean flux is vanishing because of the result of strong counter-reacting positive and negative transfers, the so-called flux-loop case, which is obviously very far from a quasi-equilibrium state (Alexakis \& Biferale 2018). Similarly, in the purely hydrodynamic case of Navier-Stokes equations, it is known that homochiral and heterochiral Fourier interactions transfer energy in opposite directions across scales, even in purely 3d homogeneous and isotropic turbulence (Waleffe 1993; Biferale et al. 2012). Such subtle refinements in the description of the energy transfer properties are interesting also from an applied point of view, opening the way for controlling both small and large scale flow behaviours by suitable forcing properties, aimed to switch on/off some energy transfer sub-channel.
In this paper we focus on the multi-scale properties of the energy transfer for 3d MHD under isotropic and homogeneous conditions. In particular, we present the results from a series of direct numerical simulations to study the response of the conducting fluid to a family of large-scale stochastic forcing mechanisms parametrised by one single parameter, $0 \leq a \leq 1$, where $a=1$ means forcing only on the kinetic channel and $a=0$ only on the magnetic one. We study the multi-scale properties of the energy transfer, using two different levels of splitting. First, we analyze the properties of the four sub-classes (channels) given by (i) the kinetic non-linear advection, (ii) the Lorentz force, (iii) the magnetic advection and (iv) magnetic stretching term. Second, we further decompose each of the four fluxes in two subchannels given by heterochiral and homochiral components in order to distinguish forward, inverse and fluxloop cascades. The main results are: (i) the mean energy transferred by the kinetic advection channel is strongly depleted as soon as we switch on a small injection on the magnetic field; (ii) this negligible mean flux is the result of a flux-loop balance between heterochiral (direct transfer) and homochiral (inverse transfer); (iii) heterochiral transfers are strongly affected by the relative amount of magnetic/kinetic forcing; (iv) we observed that the velocity-magnetic energy exchange mediated by mixed triads tends to move energy from magnetic to velocity fields.

The article is organised as follows. In Sec. 2 we present our numerical dataset. In Sec. 3 we describe the results obtained by splitting the total flux in four components and in Sec. 4 how the ones obtained after a further decomposition in heterochiral and homochiral channels. Results concerning the exchange between kinetic and magnetic fields can be found in Sec. 4.1 and the ones for small-scale fluctuations in Sec. 4.2

\section{DESCRIPTION OF THE DATASET}

To study the effect of large-scale forcing mechanisms on the multiscale properties of MHD, we conduct direct numerical simulations of the incompressible MHD equations

$$
\begin{gathered}
\frac{\partial \boldsymbol{v}}{\partial t}+(\boldsymbol{v} \cdot \nabla) \boldsymbol{v}=-\nabla p_{M}+(\boldsymbol{b} \cdot \nabla) \boldsymbol{b}+\nu \Delta \boldsymbol{v}+\sqrt{a} \boldsymbol{f}_{v} \\
\frac{\partial \boldsymbol{b}}{\partial t}+(\boldsymbol{v} \cdot \nabla) \boldsymbol{b}=(\boldsymbol{b} \cdot \nabla) \boldsymbol{v}+\eta \Delta \boldsymbol{b}+\sqrt{1-a} \boldsymbol{f}_{b} \\
\nabla \cdot \boldsymbol{v}=0, \quad \nabla \cdot \boldsymbol{b}=0
\end{gathered}
$$

where $\boldsymbol{v}, \boldsymbol{b}, p_{M}=p+|\boldsymbol{b}|^{2} / 2, \nu$ and $\eta$ denote the velocity, the magnetic field, the total pressure, the kinematic viscosity and the magnetic resistivity, respectively. 
The velocity and magnetic fields are driven respectively by $\sqrt{a} \boldsymbol{f}_{v}$ and $\sqrt{1-a} \boldsymbol{f}_{b}$ at the first two wave numbers, where $\boldsymbol{f}_{v}$ and $\boldsymbol{f}_{b}$ are random, Gaussian-distributed and $\delta(t)$-correlated forces and $\left\langle\left|\boldsymbol{f}_{v}\right|^{2}\right\rangle=\left\langle\left|\boldsymbol{f}_{b}\right|^{2}\right\rangle$. As a result the total energy input:

$$
a\left\langle\left|\boldsymbol{f}_{v}\right|^{2}\right\rangle+(1-a)\left\langle\left|\boldsymbol{f}_{b}\right|^{2}\right\rangle=\epsilon_{v}^{i n}+\epsilon_{b}^{i n}=\epsilon^{i n}=\text { const. }
$$

is independent of $a$. Modifying the parameter $a$ allows us to construct an injection mechanism from purely mechanical, $a=1$, to purely magnetic, $a=0$. A first visual understanding of our set-up can be seen in Fig. 1, where we show a $3 \mathrm{~d}$ rendering of kinetic and magnetic energy distribution in the whole volume for three characteristic values, $a=0,0.5,1$. The presence of some unbalance between purely kinetic and purely magnetic forcing is detectable even by naked eyes, our goal is to quantify and go deeper into the entangled dynamics at changing the forcing properties $a$.

From a practical point of view, we numerically solved eqs. (1)-(3) on a cubic domain $\Omega=[0,2 \pi]^{3}$ with periodic boundary conditions in all directions using the standard pseudo-spectral method with dealiasing by the two-thirds rule. The fields were advanced in time by a second-order Adam-Bashforth scheme. All runs discussed here were carried out using 512 collocation points in each direction. The simulations were initialized by supplying randomly generated velocity and magnetic field fluctuations at wavenumbers $1 \leq|\boldsymbol{k}| \leq 5$, with spectra proportional to $1 /\left[1+\left(k / k_{0}\right)^{11 / 3}\right]$ with $k_{0}=3$. Magnetic and kinetic energies were initially in equipartition in all cases, where $E_{v}=0.5$ and $E_{b}=0.5$. The magnetic Prandtl number is $\mathrm{Pm}=\nu / \eta=1$ for all simulations, and we did not impose an external mean magnetic field. The cross-helicity $\sigma_{c}=2\langle\boldsymbol{v} \cdot \boldsymbol{b}\rangle /\left\langle|\boldsymbol{v}|^{2}+|\boldsymbol{b}|^{2}\right\rangle$, though not exactly zero, is very small. So here we will be studying the energy transfer in simplified cases, lacking complexities that cross-helicity might introduce. In Fig. 2 we show the time evolution of the kinetic and magnetic energy at changing the control parameter, $a$. As one can see, all runs reached a stationary state after a time of the order of $\sim 2 T$, where $T=L_{v} / U$ is the largeeddy turnover time, $U=\sqrt{2 E_{v} / 3}$ is the r.m.s. velocity, $L_{v}=\left[\sum_{k} k^{-1} E_{v}(k)\right] / E_{v}$ is the kinetic integral length scale and $E_{v}(k)$ is the kinetic energy spectrum. Note that the large-eddy turnover times for the runs are different (see Table 1), and the time throughout the paper will be in units of the characteristic time $T$ of R1. All our analysis will be limited in the statistically stationary interval, i.e. from $t / T=6.25$ to $t / T=12.5$, where we used 31 equispaced snapshots. Further details on simulation parameters and main observables are summarised in Table 1.
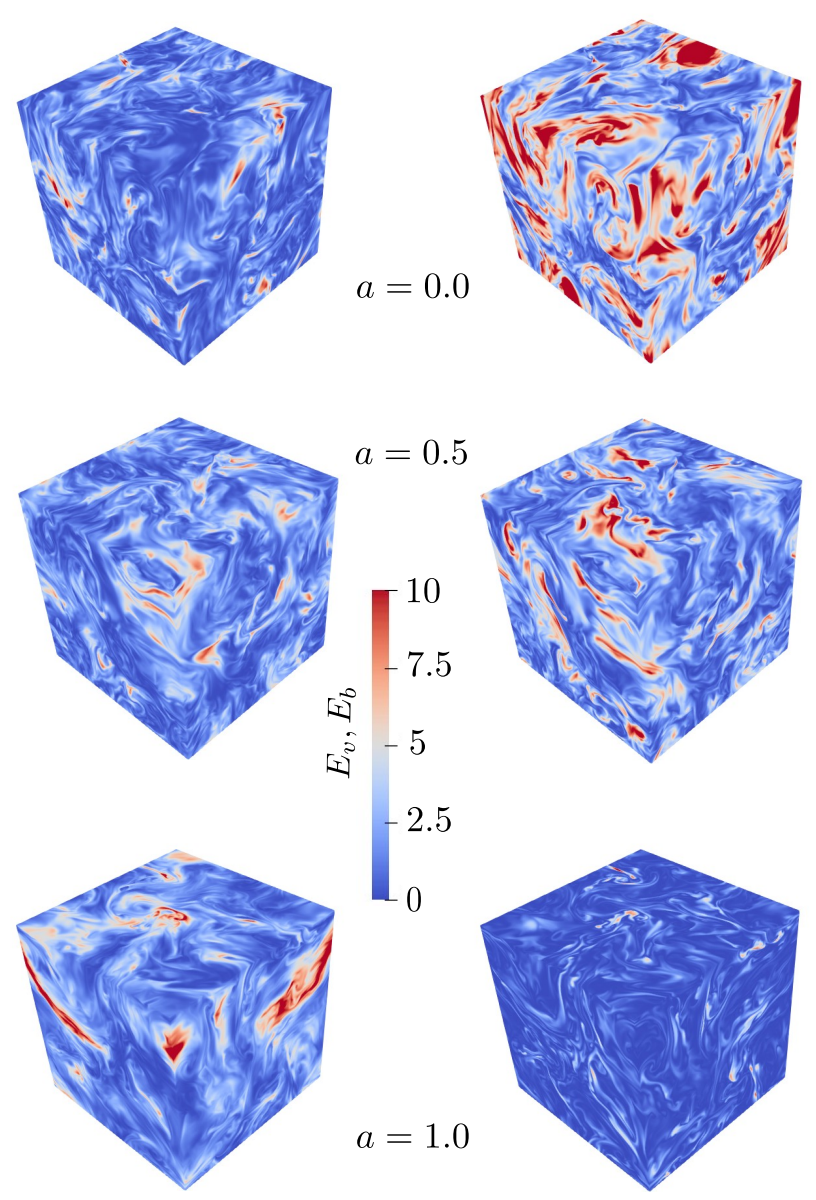

Figure 1. Visualizations of (unaveraged) kinetic (left) and (unaveraged) magnetic (right) energy distributions for $a=$ $0.00, a=0.50$ and $a=1.00$ at $t / T=6.9$.

In the inset of Fig. 2 we show also the evolution of the kinetic and magnetic Taylor-scale Reynolds numbers,

$$
\begin{aligned}
& R e_{\lambda}^{v}=\frac{U \lambda_{v}}{\nu}, \\
& R e_{\lambda}^{b}=\frac{U \lambda_{b}}{\eta},
\end{aligned}
$$

where $\lambda_{v}=U / \omega_{\mathrm{rms}}$ and $\lambda_{b}=B / j_{\mathrm{rms}}$ are the kinetic and magnetic Taylor microscales respectively, $B=\sqrt{2 E_{b} / 3}$ is the r.m.s. magnetic magnitude, and $\omega_{\text {rms }}$ and $j_{\text {rms }}$ are the rms vorticity $\omega=|\nabla \times \boldsymbol{v}|$ and current density $j=|\nabla \times \boldsymbol{b}|$ respectively. In Fig. 3 one can find the averaged values for all the above quantities, evaluated on the stationary regime as indicated by the vertical dashed lines in Fig. 2. Fig. 4 shows the kinetic and 


\begin{tabular}{ccccccccccccc}
\hline id & $N$ & $a$ & $R e_{L}^{v}$ & $R e_{L}^{b}$ & $R e_{\lambda}^{v}$ & $R e_{\lambda}^{b}$ & $E_{v}$ & $E_{b}$ & $\varepsilon_{v}$ & $\varepsilon_{b}$ & $T$ & $k_{\max } \eta_{v}$ \\
\hline $\mathrm{R} 1$ & 512 & 0.00 & 1663 & 4506 & 105 & 141 & 1.69 & 3.87 & 0.87 & 1.10 & 2.40 & 1.65 \\
$\mathrm{R} 2$ & 512 & 0.20 & 1665 & 3366 & 105 & 127 & 1.64 & 3.14 & 0.82 & 1.07 & 2.47 & 1.68 \\
$\mathrm{R} 3$ & 512 & 0.25 & 1675 & 3317 & 105 & 126 & 1.67 & 3.16 & 0.84 & 1.10 & 2.44 & 1.67 \\
$\mathrm{R} 4$ & 512 & 0.30 & 1694 & 3224 & 106 & 128 & 1.63 & 2.92 & 0.79 & 1.03 & 2.54 & 1.70 \\
$\mathrm{R} 5$ & 512 & 0.40 & 1802 & 2865 & 109 & 122 & 1.69 & 2.81 & 0.80 & 1.07 & 2.59 & 1.69 \\
$\mathrm{R} 6$ & 512 & 0.50 & 2081 & 2721 & 117 & 122 & 1.83 & 2.72 & 0.82 & 1.12 & 2.75 & 1.68 \\
$\mathrm{R} 7$ & 512 & 0.70 & 2244 & 2247 & 122 & 115 & 1.88 & 2.42 & 0.80 & 1.16 & 2.90 & 1.69 \\
$\mathrm{R} 8$ & 512 & 0.75 & 2376 & 2269 & 125 & 116 & 1.94 & 2.43 & 0.80 & 1.17 & 2.99 & 1.69 \\
$\mathrm{R} 9$ & 512 & 0.80 & 2585 & 1731 & 131 & 107 & 1.96 & 2.02 & 0.75 & 1.16 & 3.21 & 1.72 \\
$\mathrm{R} 10$ & 512 & 0.90 & 2716 & 1630 & 134 & 104 & 2.00 & 2.01 & 0.75 & 1.24 & 3.29 & 1.72 \\
$\mathrm{R} 11$ & 512 & 0.95 & 3148 & 1162 & 144 & 94 & 2.04 & 1.60 & 0.68 & 1.24 & 3.72 & 1.76 \\
$\mathrm{R} 12$ & 512 & 1.00 & 6486 & 427 & 207 & 72 & 2.60 & 0.78 & 0.53 & 1.30 & 6.07 & 1.87 \\
\hline
\end{tabular}

Table 1. Parameters and key observable for all simulations: grid size $N^{3}$, forcing parameter $a$, kinetic and magnetic integralscale Reynolds numbers $R e_{L}^{v}=U L_{v} / \nu$ and $R e_{L}^{b}=U L_{b} / \eta$ where $U=\sqrt{2 E_{v} / 3}$ is the rms velocity and $L_{v}=\left[\sum_{k} k^{-1} E_{v}(k)\right] / E_{v}$ and $L_{b}=\left[\sum_{k} k^{-1} E_{b}(k)\right] / E_{b}$ are the kinetic and magnetic integral length scales respectively, kinetic and magnetic Taylorscale Reynolds numbers $R e_{\lambda}^{v}=U \lambda_{v} / \nu$ and $R e_{\lambda}^{b}=U \lambda_{b} / \eta$ where $\lambda_{v}$ and $\lambda_{b}$ are the kinetic and magnetic Taylor microscales respectively, kinetic energy $E_{v}$, magnetic energy $E_{b}$, kinetic dissipation rate $\varepsilon_{v}$, magnetic dissipation rate $\varepsilon_{b}$, large-eddy turnover time $T=L_{v} / U$, the largest resolved wavenumber $k_{\max }=N / 3$, and kinetic Kolmogorov microscale $\eta_{v}=\left(\nu^{3} / \varepsilon_{v}\right)^{1 / 4}$. All observables are time averaged. 

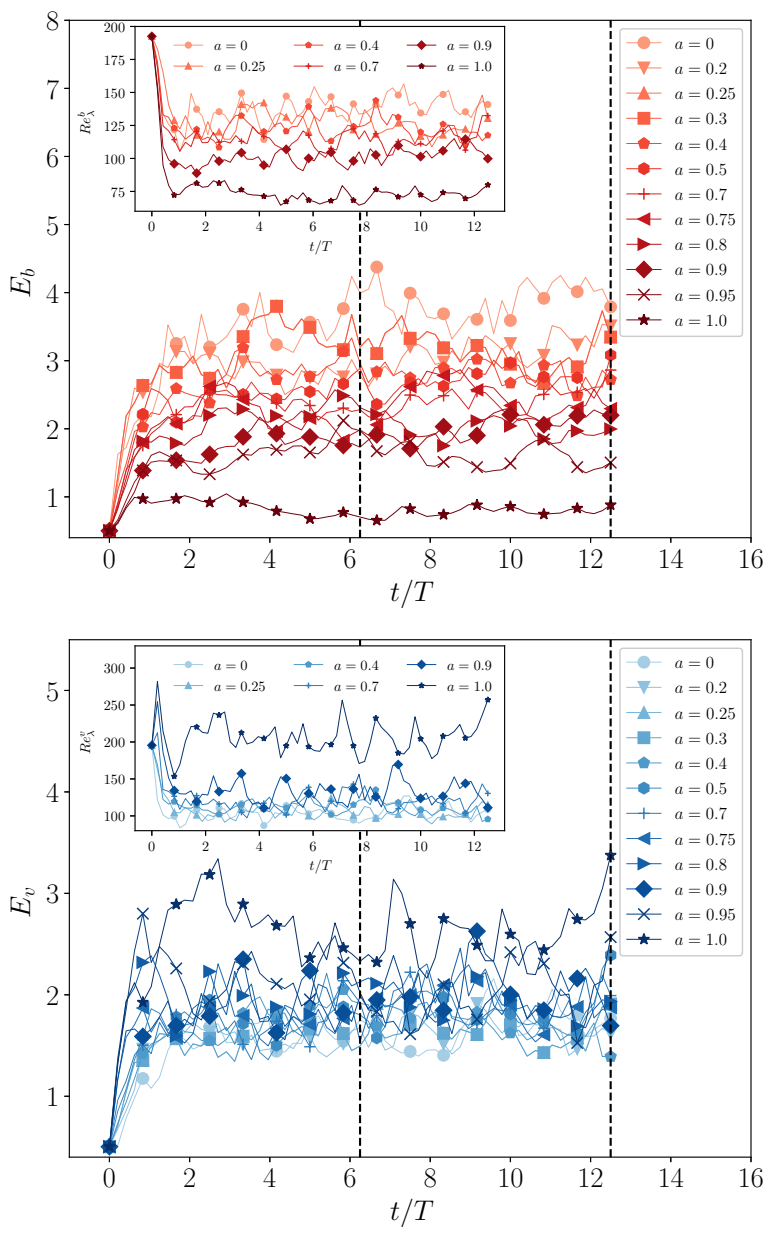

Figure 2. Time evolution of magnetic (top) and kinetic (bottom) energies as functions of $a$. Insets: time evolution of the Taylor-scale Reynolds numbers as functions of $a$ are shown in the insets. The interval used in the data analyses is indicated by the vertical lines.

magnetic energy spectra

$$
\begin{aligned}
& E_{v}(k)=\frac{1}{2} \sum_{|\boldsymbol{k}|=k}\left\langle|\hat{\boldsymbol{v}}(\boldsymbol{k}, t)|^{2}\right\rangle_{t}, \\
& E_{b}(k)=\frac{1}{2} \sum_{|\boldsymbol{k}|=k}\left\langle|\hat{\boldsymbol{b}}(\boldsymbol{k}, t)|^{2}\right\rangle_{t},
\end{aligned}
$$

where $\langle\cdots\rangle_{t}$ denotes a time average over 31 snapshots and $\hat{x}$ the Fourier transform.

As can be seen from the spectra, the variation of $a$ is mostly visible at the large magnetic scales, while the large-scale dynamics of the velocity field is weakly affected, at least at the level of energy spectra. The kinetic energy spectrum shows some difference in the inertialrange scaling and amplitude. For both magnetic and kinetic energy spectra, we note that $a=1.00$, that is, fully mechanical forcing is quasi-singular in appearance, in the sense that significant differences in the spectra are

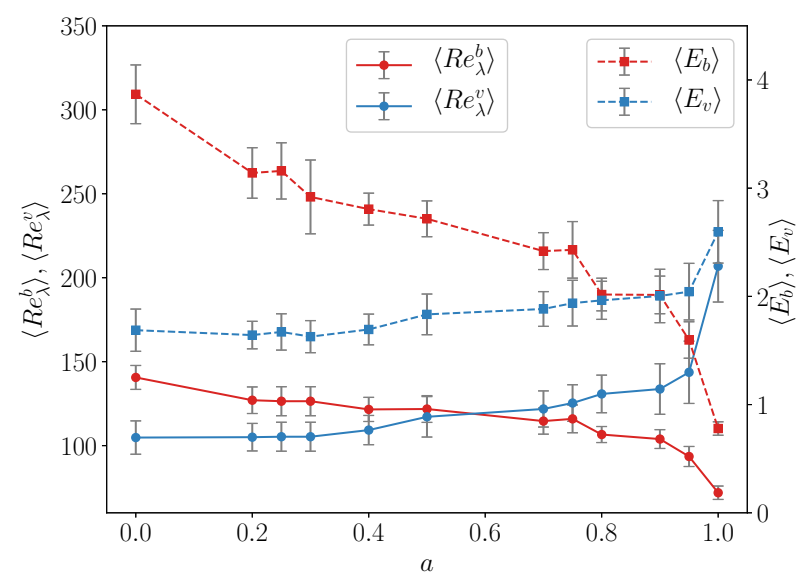

Figure 3. Time-averaged Taylor-scale Reynolds numbers and magnetic and kinetic energies as functions of $a$. The error bars show the standard error.

discernible between $a=1.00$ and $a=0.95$. For smaller values of $a$ the spectra show little variation. The overall picture emerging from these few quantitative results confirm what already said in the previous text: it is very difficult, if not impossible, to disentangle the intricate multi-scale properties of the conducting flow by looking only at mean global properties based on spectra. In order to overcome this limitation, in the following section we will introduce the multi-channel flux decomposition as anticipated in the introduction.

\section{FLUX DECOMPOSITION: KINETIC, LORENTZ, MAGNETIC ADVECTION, MAGNETIC STRETCHING}

In order to study the multi-scale energy dynamics all vector fields must be decomposed into large and smallscale components. To do so, we apply a filtering operation to all terms in eqs. (1) and (2) (Zhou \& Vahala 1991; Kessar et al. 2016; Yang et al. 2016; Aluie 2017; Offermans et al. 2018). In what follows, we briefly summarise the main procedure, for the derivations and further details see (Kessar et al. 2016; Aluie 2017; Offermans et al. 2018). Given a filter kernel $G^{\ell}$, the filtered component of a square-integrable function $\varphi(\boldsymbol{x})$ in the domain $\Omega$, is defined as

$\bar{\varphi}^{\ell}(\boldsymbol{x}) \equiv \int_{\Omega} d \boldsymbol{y} G^{\ell}(\boldsymbol{x}-\boldsymbol{y}) \varphi(\boldsymbol{y})=\sum_{\boldsymbol{k} \in \mathbb{Z}^{3}} \hat{G}^{\ell}(\boldsymbol{k}) \hat{\varphi}(\boldsymbol{k}) e^{i \boldsymbol{k} \boldsymbol{x}}$,

where $\ell$ is the filter width. Several choices of $G$, such as Gaussian filters, top hat filters or Galerkin projectors may be used to decompose a given function into largescale and small-scale components. For the present study, 

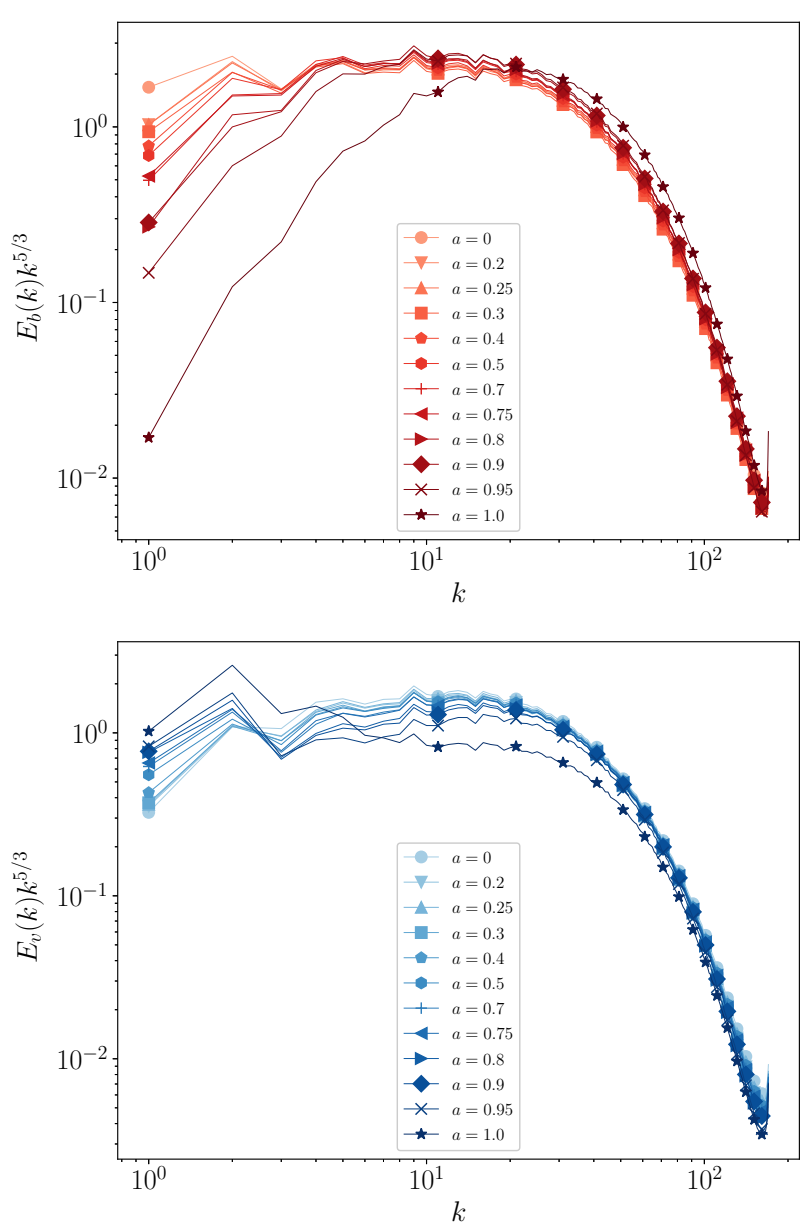

Figure 4. Magnetic (top) and kinetic (bottom) compensated energy spectra for different values of $a$, where $a=1.00$ corresponds to fully mechanical forcing and $a=0.00$ to fully electromagnetic forcing. The spectra have been averaged over 31 configurations during the statistically stationary evolution.

$G^{\ell}$ is a spherically symmetric Galerkin projector

$$
\hat{G}^{\ell}(\boldsymbol{k})= \begin{cases}1 & \text { if }|\boldsymbol{k}| \leqslant k_{c} \\ 0 & \text { if }|\boldsymbol{k}|>k_{c}\end{cases}
$$

where $k_{c}=\pi / \ell$. Galerkin projectors have a number of advantages and disadvantages. On the positive side, the projection operation results in a clear distinction between sub-filter and resolved scales, and the sub-filterscale energy fluxes calculated with respect to the projector coincide exactly with the classically defined Fourier fluxes. Drawbacks are mainly concerned with filterinduced oscillations in configuration space that result in sub-filter-scale stresses that are not positive definite (Vreman et al. 1994). However, the statistics of the subfilter-scale energy transfer calculated through Gaussian smoothing or Galerkin projection vary little for NavierStokes turbulence (Buzzicotti et al. 2018).

The filtered MHD equations read

$$
\begin{aligned}
\partial_{t} \bar{v}_{i}^{\ell}= & -\partial_{j}\left({\overline{\bar{v}_{i}^{\ell} \bar{v}_{j}^{\ell}}}^{\ell}-\overline{\bar{b}_{i}^{\ell} \bar{b}_{j}^{\ell}}+\tau_{i j}^{\ell, I}-\tau_{i j}^{\ell, M}+\bar{p}^{\ell} \delta_{i j}\right) \\
& +\nu \partial_{j j} \bar{v}_{i}^{\ell}+\sqrt{a}{\overline{f_{v i}}}^{\ell} \\
\partial_{t} \bar{b}_{i}^{\ell}= & -\partial_{j}\left(\overline{\bar{b}_{i}^{\ell} \bar{v}_{j}^{\ell}}-\overline{\bar{v}_{i}^{\ell} \bar{b}_{j}^{\ell}}+\tau_{i j}^{\ell, A}-\tau_{i j}^{\ell, D}\right) \\
& +\eta \partial_{j j} \bar{b}_{i}^{\ell}+\sqrt{1-a}{\overline{f_{b i}}}^{\ell}
\end{aligned}
$$

where we sum over repeated indices and

$$
\begin{aligned}
\tau_{i j}^{\ell, I} & ={\overline{v_{i} v_{j}}}^{\ell}-{\overline{\bar{v}_{i}^{\ell} \bar{v}_{j}^{\ell}}}^{\ell}, \\
\tau_{i j}^{\ell, M} & ={\overline{b_{i} b_{j}}}^{\ell}-\overline{\bar{b}}_{i}^{\ell} \bar{b}_{j}^{\ell}, \\
\tau_{i j}^{\ell, A} & ={\overline{b_{i} v_{j}}}^{\ell}-\overline{\bar{b}}_{i}^{\ell} \bar{v}_{j}^{\ell} \\
\tau_{i j}^{\ell, D} & ={\overline{v_{i} b_{j}}}^{\ell}-\overline{\bar{v}_{i}^{\ell} \bar{b}_{j}^{\ell}},
\end{aligned}
$$

denote the inertial (I), Maxwell (M), advective (A) and dynamo (D) subfilter-scale stresses, respectively. Despite their common origin through the electric field in the induction equation, we here treat $\tau_{i j}^{\ell, A}$ and $\tau_{i j}^{\ell, D}$ separately, in order to disentangle the effects of magneticfield-line advection, encoded in $\tau_{i j}^{\ell, A}$, and magnetic-fieldline stretching, encoded in $\tau_{i j}^{\ell, D}$. Usually, the magnetic sub-scale stress refers to the difference $\tau_{i j}^{\ell, A}-\tau_{i j}^{\ell, D}$ (Aluie 2017; Offermans et al. 2018). Equations (11) and (12) differ from expressions for the filtered MHD equations found elsewhere by an additional projection of the coupling terms. The latter ensures that the dynamics defined by eqs. (11) and (12) are confined to the same finite-dimensional subspace $\Omega^{\ell}$ of the original domain $\Omega$ (Buzzicotti et al. 2018; Offermans et al. 2018). At first sight, this formulation suggests that the corresponding evolution equations for kinetic and magnetic energy feature terms that are not Galilean invariant, which ought to be avoided as the measured subfilter-scale energy transfers otherwise include unphysical fluctuations (Aluie \& Eyink 2009a,b; Buzzicotti et al. 2018). However, the energy balance equations can be expressed in an alternative way by including terms that vanish under spatial averaging and ensure Galilean invariance of all terms (Buzzicotti et al. 2018; Offermans et al. 2018). For a statistically stationary evolution, the spatio-temporally averaged energy budget can then be written as

$$
\begin{aligned}
\sqrt{a}\left\langle\bar{v}_{i}^{\ell} \overline{f_{v i}}\right\rangle & =\Pi_{I}^{\ell}-\Pi_{M}^{\ell}-\pi_{M}^{\ell}+\varepsilon_{v}^{\ell}, \\
\sqrt{1-a}\left\langle\bar{b}_{i}^{\ell} \overline{f_{b i}} \ell\right. & =\Pi_{A}^{\ell}-\Pi_{D}^{\ell}-\pi_{D}^{\ell}+\varepsilon_{b}^{\ell},
\end{aligned}
$$


where $\varepsilon_{v}^{\ell}=\nu\left\langle\partial_{j} \bar{v}_{i}^{\ell} \partial_{j} \bar{v}_{i}^{\ell}\right\rangle$ and $\varepsilon_{b}^{\ell}=\eta\left\langle\partial_{j} \bar{b}_{i}^{\ell} \partial_{j} \bar{b}_{i}^{\ell}\right\rangle$ are the filtered kinetic and magnetic dissipation rates, respectively, and $\pi_{D}^{\ell}=-\left\langle\left(\partial_{j} \bar{b}_{i}^{\ell}\right) \bar{v}_{i}^{\ell} b_{j}^{\ell}\right\rangle=\left\langle\left(\partial_{j} \bar{v}_{i}^{\ell}\right) \bar{b}_{i}^{\ell} \bar{b}_{j}^{\ell}\right\rangle=$ $-\pi_{M}^{\ell}$, are terms that convert kinetic to magnetic en$\operatorname{ergy}\left(\pi_{D}^{\ell}\right)$ and vice versa $\left(\pi_{M}^{\ell}\right)$, and

$$
\begin{aligned}
\Pi_{I}^{\ell} & =-\left\langle\left(\partial_{j} \bar{v}_{i}^{\ell}\right) \tau_{i j}^{\ell, I}\right\rangle, \\
\Pi_{M}^{\ell} & =-\left\langle\left(\partial_{j} \bar{v}_{i}^{\ell}\right) \tau_{i j}^{\ell, M}\right\rangle, \\
\Pi_{A}^{\ell} & =-\left\langle\left(\partial_{j} \bar{b}_{i}^{\ell}\right) \tau_{i j}^{\ell, A}\right\rangle, \\
\Pi_{D}^{\ell} & =-\left\langle\left(\partial_{j} \bar{b}_{i}^{\ell}\right) \tau_{i j}^{\ell, D}\right\rangle,
\end{aligned}
$$

denote the four proper energy fluxes, in the sense that they vanish in the limit $\ell \rightarrow 0$, as can be seen from eqs. (13)-(16). If positive, the inertial and Maxwell fluxes, $\Pi_{I}^{\ell}$ and $-\Pi_{M}^{\ell}$ transfer kinetic energy from scales larger than or equal to $\ell$ to scales smaller than $\ell$ and vice versa if negative, while the advective and dynamo fluxes, $\Pi_{A}^{\ell}$ and $-\Pi_{D}^{\ell}$, do so with magnetic energy. Note that there is no interscale energy conversion as the conversion terms $\pi_{M}^{\ell}$ and $\pi_{D}^{\ell}$ only involve filtered fields, as such they are known as resolved-scale conversion terms (Aluie 2017). The total energy flux is then given by the sum

$$
\Pi^{\ell}=\underbrace{-\pi_{D}^{\ell}-\pi_{M}^{\ell}}_{=0 \forall \ell}+\Pi_{I}^{\ell}-\Pi_{M}^{\ell}+\Pi_{A}^{\ell}-\Pi_{D}^{\ell} .
$$

\subsection{Flux decomposition: numerical results}

In this section we start by looking at the response of the four energy fluxes (19-22) at changing the forcing input parameter, $a$, as shown in Fig. 5. As one can see, the first important result is given by the net transition of $\Pi_{I}^{\ell}$ when $a<1$, where the total energy transfer along this channel becomes almost vanishing at all scales (top left panel). A depletion of the inertial flux to almost zero in MHD turbulence subject to partially magnetic large-scale forcing had already been observed by Alexakis (2013) at $R e_{\lambda}^{v} \approx 300$ for $a=0.8$ and $a \approx 0.86$. In both cases the magnetic forcing was helical, with a relative helicity $\rho=0.31$ for $a=0.8$ and $\rho=0.5$ for $a \approx 0.86$. An injection of magnetic helicity and the subsequent local-in-scale generation of kinetic helicity (Linkmann et al. 2017) lead to a depletion of nonlinearity close to the injection scale. Here, both forcing functions are non-helical, hence the combination of the calculations by Alexakis (2013) with those reported here suggest that the depletion of the inertial flux is a generic feature of magnetically forced MHD turbulence, irrespective of helicity injection. As a result, the energy injected by the large-scale forcing on the velocity field is transferred to small scales only via the Lorentz contribution, i.e. the $-\Pi_{M}^{\ell}$ term (top right panel), which is pretty independent on the $a$ value. The sign conventions for $\Pi_{M}^{\ell}$ and $\Pi_{D}^{\ell}$ reflect signs which occur in eqs. (17) and (18). For $a \leqslant 0.5$ (magnetically dominated forcing) an inertial range in $\Pi_{A}^{\ell}$ develops, indicating a direct cascade of magnetic fluctuations, see bottom left panel of Fig. 5, typical of the physics of a passive vector advected by a turbulent flow and a signature that the direct contribution of the forcing on the induction equation introduces an important 'linear' component in the magnetic transfer (Kraichnan 1994; Vergassola 1996; Chertkov et al. 1999). At these values of $a$ the inertial transfer $\Pi_{I}^{\ell}$ is very depleted despite the large-scale conversion of $b \rightarrow v$, see top left panel of Fig. 5. The marked change for $a<1$ have also a clear counterpart in the spectral scaling as seen by comparison of the energy spectra shown in Fig. 4 between $a=0.95$ and $a=1.00$. The dynamo contribution $-\Pi_{D}^{\ell}$ (bottom right panel) does not develop any clear systematic plateau across wavenumbers. Its tendency to be more active at smaller and smaller $k$ by decreasing $a$ might results from the effect of increasing magnetic energy injection at large scales.

\section{HOMOCHIRAL AND HETEROCHIRAL SUB-CHANNELS}

Any solenoidal vector field can be decomposed in positively and negatively helical components (Waleffe 1992):

$$
\begin{aligned}
& \boldsymbol{v}(\boldsymbol{x}, t)=\boldsymbol{v}^{+}(\boldsymbol{x}, t)+\boldsymbol{v}^{-}(\boldsymbol{x}, t) \\
& \boldsymbol{b}(\boldsymbol{x}, t)=\boldsymbol{b}^{+}(\boldsymbol{x}, t)+\boldsymbol{b}^{-}(\boldsymbol{x}, t)
\end{aligned}
$$

where

$$
\begin{aligned}
\boldsymbol{v}^{ \pm}(\boldsymbol{x}, t) & \equiv \sum_{\boldsymbol{k}}\left(\hat{\boldsymbol{v}}_{\boldsymbol{k}}(t) \cdot \boldsymbol{h}_{\boldsymbol{k}}^{ \pm}\right) \boldsymbol{h}_{\boldsymbol{k}}^{ \pm} e^{i \boldsymbol{k} \cdot \boldsymbol{x}} \\
\boldsymbol{b}^{ \pm}(\boldsymbol{x}, t) & \equiv \sum_{\boldsymbol{k}}\left(\hat{\boldsymbol{b}}_{\boldsymbol{k}}(t) \cdot \boldsymbol{h}_{\boldsymbol{k}}^{ \pm}\right) \boldsymbol{h}_{\boldsymbol{k}}^{ \pm} e^{i \boldsymbol{k} \cdot \boldsymbol{x}}
\end{aligned}
$$

where $\boldsymbol{h}_{\boldsymbol{k}}^{ \pm}$are eigenfunctions of the curl operator $i \boldsymbol{k} \times(\cdot)$ with eigenvalues \pm 1 . As a result, plugging the decomposition (24-25) in (11-12) one gets different expressions for the spatio-temporally averaged energy sub-fluxes which can be reduced to homochiral or heterochiral contributions, depending whether the three fields entering in the expressions (19-22) have all the same chirality or there is one with opposite chirality of the other two (for details see Appendix A). As a result, the energy balance across 

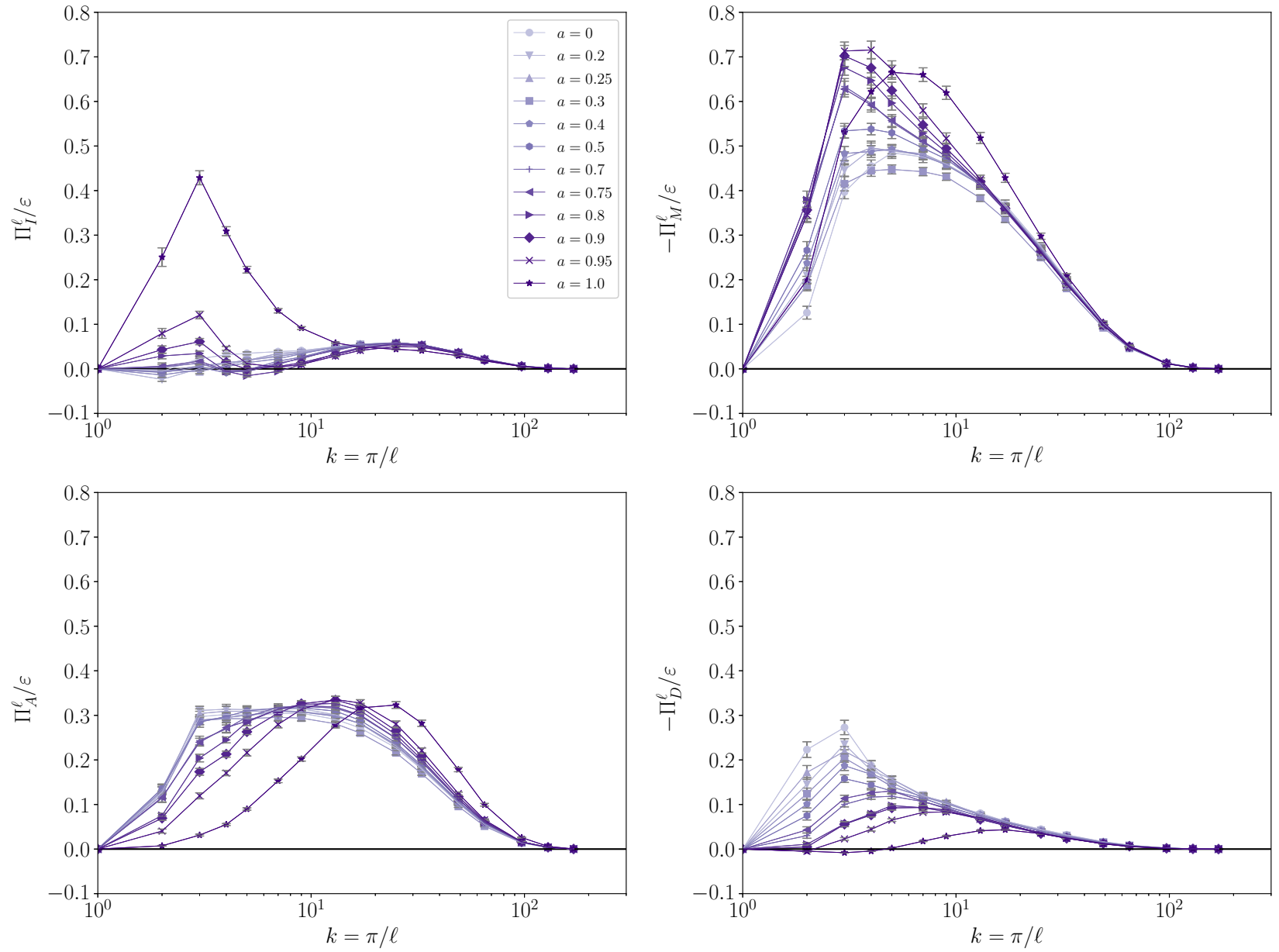

Figure 5. Decomposed fluxes for different values of $a$, normalized with the total energy dissipation rate $\varepsilon=\varepsilon_{v}+\varepsilon_{b}$. Top left: $\Pi_{I}^{\ell}$, top right: $-\Pi_{M}^{\ell}$, bottom left: $\Pi_{A}^{\ell}$, bottom right: $-\Pi_{D}^{\ell}$. The decreasing color gradient indicates decreasing values of $a$. The error bars show the standard error. 
scale can be further refined to:

$$
\begin{aligned}
& \sqrt{a}\left\langle\bar{v}_{i}^{\ell}{\overline{f_{v i}}}^{\ell}\right\rangle=\left(\Pi_{I}^{h o, \ell}+\Pi_{I}^{h e, \ell}\right)-\left(\Pi_{M}^{h o, \ell}+\Pi_{M}^{h e, \ell}\right) \\
& -\left(\pi_{M}^{h o, \ell}+\pi_{M}^{h e, \ell}\right)+\varepsilon_{v}^{\ell}, \\
& \sqrt{1-a}\left\langle\bar{b}_{i}^{\ell} \overline{f_{b i}} \ell=\left(\Pi_{A}^{h o, \ell}+\Pi_{A}^{h e, \ell}\right)-\left(\Pi_{D}^{h o, \ell}+\Pi_{D}^{h e, \ell}\right)\right. \\
& -\left(\pi_{D}^{h o, \ell}+\pi_{D}^{h e, \ell}\right)+\varepsilon_{b}^{\ell} .
\end{aligned}
$$

It is important to stress that all ח-labelled components are real fluxes, in the same sense of above, i.e. they vanish for $\ell \rightarrow 0$. In Fig. 6 we show the helical decomposition of the four sub-fluxes shown in Fig. 5. By looking at all four panels of Fig. 6, we see that the heterochiral components tends to decrease for smaller and smaller $a$, except for the dynamo case $\Pi_{D}^{\ell}$. Concerning the kinetic inertial channel, $\Pi_{I}^{\ell}$ (top left), it is important to observe that even in the presence of a vanishing global contribution (top left panel of Fig. 5) the dynamic is far from being close to equilibrium, with a good balancing between heterochiral contributions (forward flux) and homochiral ones (backward flux). We are in the presence of a flux-loop cascade picture for this channel (Alexakis $\&$ Biferale 2018). For the other two channels, $\Pi_{M}^{\ell}$ and $\Pi_{A}^{\ell}$ we do not observe any change in the flux direction with the heterochiral channel consistently more (less) important than the homochiral for $\Pi_{M}^{\ell}\left(\Pi_{A}^{\ell}\right)$. Finally, for $\Pi_{D}^{\ell}$ we observe that the homochiral sector is about $5 \%$ of total $\Pi^{\ell}$, with a little variance with $a$ and the heterochiral is strongly depleted with increasing $a$.

\subsection{Kinetic-magnetic conversion terms}

The total conversion of kinetic to magnetic energy is quantified by the conversion term $\pi_{D}^{\ell}=-\pi_{M}^{\ell}$ in the limit $\ell \rightarrow 0$, in which eqs. (17) and (18) become

$$
\begin{aligned}
\pi_{D}^{0} & =\sqrt{a}\left\langle\bar{v}_{i}^{0}{\overline{f_{v i}}}^{0}\right\rangle-\varepsilon_{v}, \\
-\pi_{D}^{0} & =\sqrt{1-a}\left\langle\bar{b}_{i}^{0}{\overline{f_{b i}}}^{0}\right\rangle-\varepsilon_{b},
\end{aligned}
$$

as all terms in eqs. (17) and (18) are continuous in $\ell$ and the flux terms vanish in the limit $\ell \rightarrow 0$. For the extreme cases, that is fully mechanical forcing, $a=1$, and fully electromagnetic forcing, $a=0$, one obtains

$$
\pi_{D}^{0}=\left\{\begin{array}{cc}
\varepsilon^{i n}-\varepsilon_{v}>0 & \text { for } a=1, \\
-\varepsilon^{i n}+\varepsilon_{b}<0 & \text { for } a=0,
\end{array}\right.
$$

where $\varepsilon^{i n}$ is the spatio-temporally averaged total energy injection rate, which must equal the total dissipation during statistically stationary evolution, that is $\varepsilon^{i n}=\varepsilon_{v}+\varepsilon_{b}$. Hence, and as could have been expected, the energy conversion term will change sign as a function of $a$. If the forcing is fully mechanical, the magnetic field can only be maintained by dynamo action, that is, conversion of kinetic to magnetic energy encoded by $\pi_{D}^{0}>0$. For fully electromagnetic forcing, it is the other way round, the flow is maintained turbulent by the Lorentz force and $\pi_{M}^{0}=-\pi_{D}^{0}>0$. In Fig. 7 (top panel) we show the total undecomposed conversion term $\pi_{D}^{\ell}$ at changing $a$. As one can see, while for mostly kinetic driving the conversion is finished at mid-scale (as also shown by Bian \& Aluie (2019)), for magnetic forcing the conversion appears to be maximised at a much larger scale, as can be seen from the fact that $\pi_{D}^{\ell}$ becomes almost constant for lower values of $k$, but in total weaker than the $v \rightarrow b$ dynamo $(a=1)$. As seen from the homochiral and heterochiral contributions shown in the bottom panel, on average the homochiral channel converts kinetic into magnetic energy and the heterochiral one does the opposite. Finally, in the inset of the top panel we show the global conversion term, $\pi_{D}^{\ell}$ with $\ell=\pi / k_{\max }$ as a function of $a$, showing that the main driving mechanisms upon changing the forcing properties is linked to the heterochiral triads, which change quickly as soon as $a<1$. The relative magnitudes of the different energy transfer and conversion terms for $a=0, a=0.5$ and $a=1$ are visually summarised in form of Sankey diagrams in figs. 8 and 9. The former corresponds to the decomposition of the total energy flux as in eq. (23) and the latter includes the helically decomposed terms as in eqs. (28) and (29). As can be seen in Fig. 9, the dynamo is always active, even for the case of purely magnetic forcing, as the homochiral interactions convert kinetic to magnetic energy for all values of $a$ through $\pi_{D}^{h o, \ell}$ shown in Fig. 9 in light purple, see also Fig. 7. The heterochiral conversion term $\pi_{D}^{h e, \ell}$ shown in Fig. 9 in dark purple, changes its role. For $a=1$, the magnetic field is sustained by the velocity field alone through both $\pi_{D}^{h o, \ell}$ and $\pi_{D}^{h e, \ell}$, with decreasing $a$ the dynamo operates alongside the mean largescale conversion of magnetic to kinetic energy due to the Lorentz force, which is associated with heterochiral interactions, that is with $\pi_{D}^{h e, \ell}$, for $a \neq 1$. The resulting resolved-scale transfer loop formed $\pi_{D}^{h o, \ell}$ and $\pi_{D}^{h e, \ell}$ is clearly visible in fig. 9 for $a=0$ and $a=0.5$. Interestingly, and in contrast to the dynamo for $a=1$, the mean energy transfer due to the Lorentz force does vanish in both homo- and heterochiral transfer components $\pi_{D}^{h o, \ell}$ and $\pi_{D}^{h e, \ell}$ separately for $a=0$.

The relation of homo- and heterochiral triads to energy transfer and conversion in Navier-Stokes and MHD turbulence has been studied through shell models (Lessinnes et al. 2009; De Pietro et al. 2015; Rathmann 

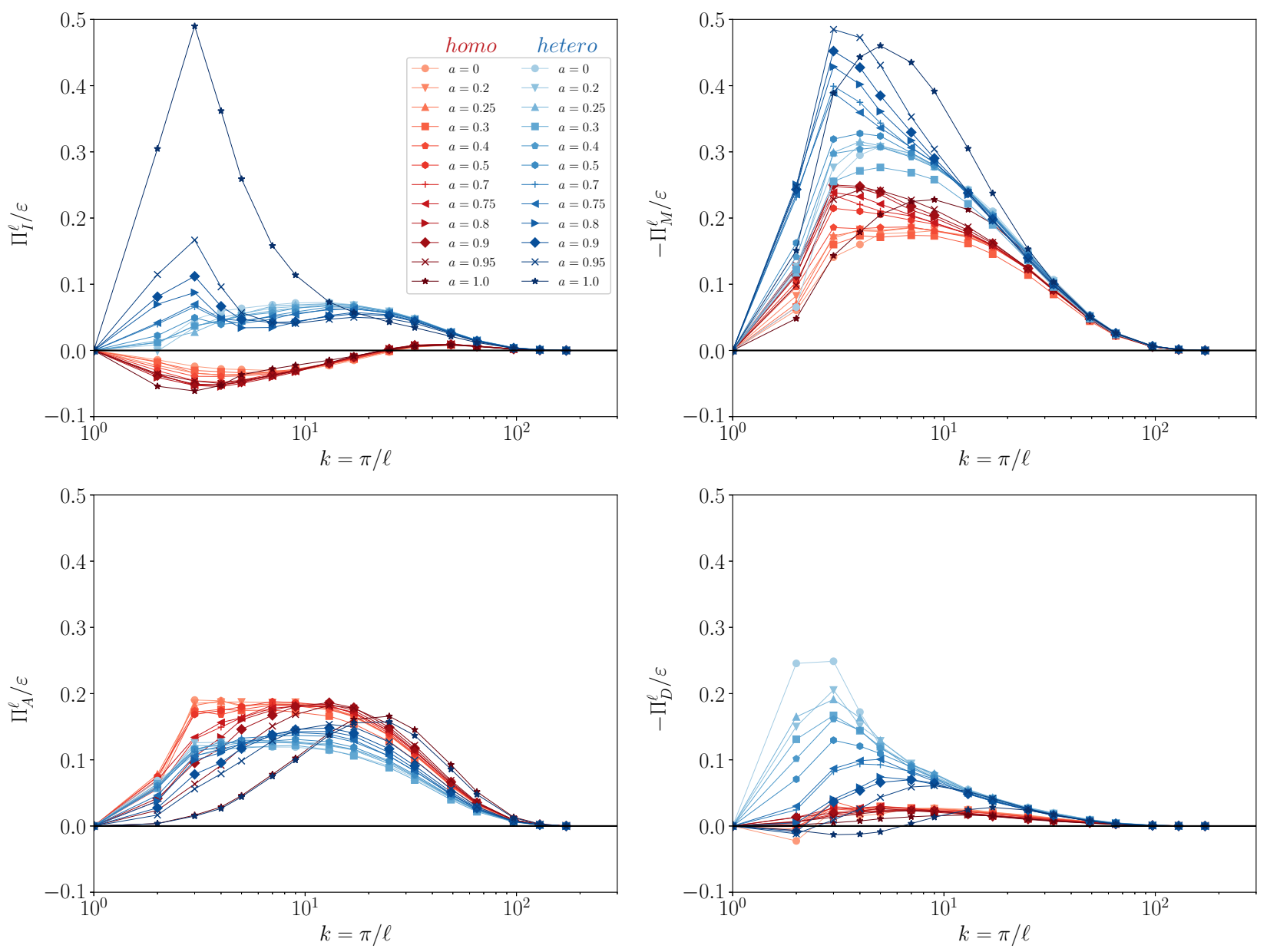

Figure 6. Hetero- and homochiral fluxes for different values of $a$. 
\& Ditlevsen 2017, 2019), analytical means (Waleffe 1992; Linkmann et al. 2016, 2017), specifically designed DNS (Biferale et al. 2012; Linkmann et al. 2017) and postprocessing of DNS data (Alexakis 2017). In particular in connection with kinematic dynamo action, analytical results based on stability analyses of equilibria of minimal representations of the MHD equations obtained by Galerkin truncation suggest that homochiral triads do not contribute to the conversion of kinetic to magnetic energy in the kinematic regime (Linkmann et al. 2016, 2017), both at large and small scale. Only heterochiral triads were found to contribute to the kinematic dynamo, which for a large-scale dynamo is commensurate with the helical signature of the $\alpha$-effect (Steenbeck et al. 1966; Brandenburg 2001). Numerical results, obtained by projecting the velocity field on the positively helical subspace corroborate these findings, as the different helical sectors of the magnetic field mostly grow according to the helical signature of a stretch-twist-fold dynamo (Linkmann et al. 2017). Similar results have recently been reported using specifically designed shell models of homo- or heterochiral triads and theoretical arguments based on the conservation of enstrophy-like invariants (Rathmann \& Ditlevsen 2019). Interestingly, Rathmann \& Ditlevsen (2019) found that for homochiral triads the magnetic field could not be maintained by dynamo action alone, the magnetic dynamics had to be excited by electromagnetic forcing, which confirms again that homochiral triads cannot amplify a magnetic seed field.

In summary, in the kinematic regime Linkmann et al. (2016, 2017) and Rathmann \& Ditlevsen (2019) report the opposite helical signature of that seen here for the fully nonlinear case $(a=1)$. In our nonlinear regime, the minimal homochiral Galerkin models do contain instabilities connected with dynamo action, which are not discussed in (Linkmann et al. 2016, 2017). The above discussion suggests that the process of dynamo saturation may be connected with a change in the behaviour of homo- and heterochiral triads.

\subsection{Small-scale response}

In Fig. 10 we summarise some results concerning the issue of universality of small-scales fluctuations. In the top panel we show the value of the second order moment for one component of the longitudinal gradient for both velocity and magnetic fields, $\left\langle S_{v_{i}}^{2}\right\rangle=\left\langle\left(\partial_{i} v_{i}\right)^{2}\right\rangle$ and $\left\langle S_{b_{i}}^{2}\right\rangle=\left\langle\left(\partial_{i} b_{i}\right)^{2}\right\rangle$. As one can see, the main effect is given by an increase of the total magnitude of velocity gradients by decreasing $a$, indicating that the the depletion of the kinetic channel shown in Fig. 5 does not have a direct effect on the small-scale velocity activity.
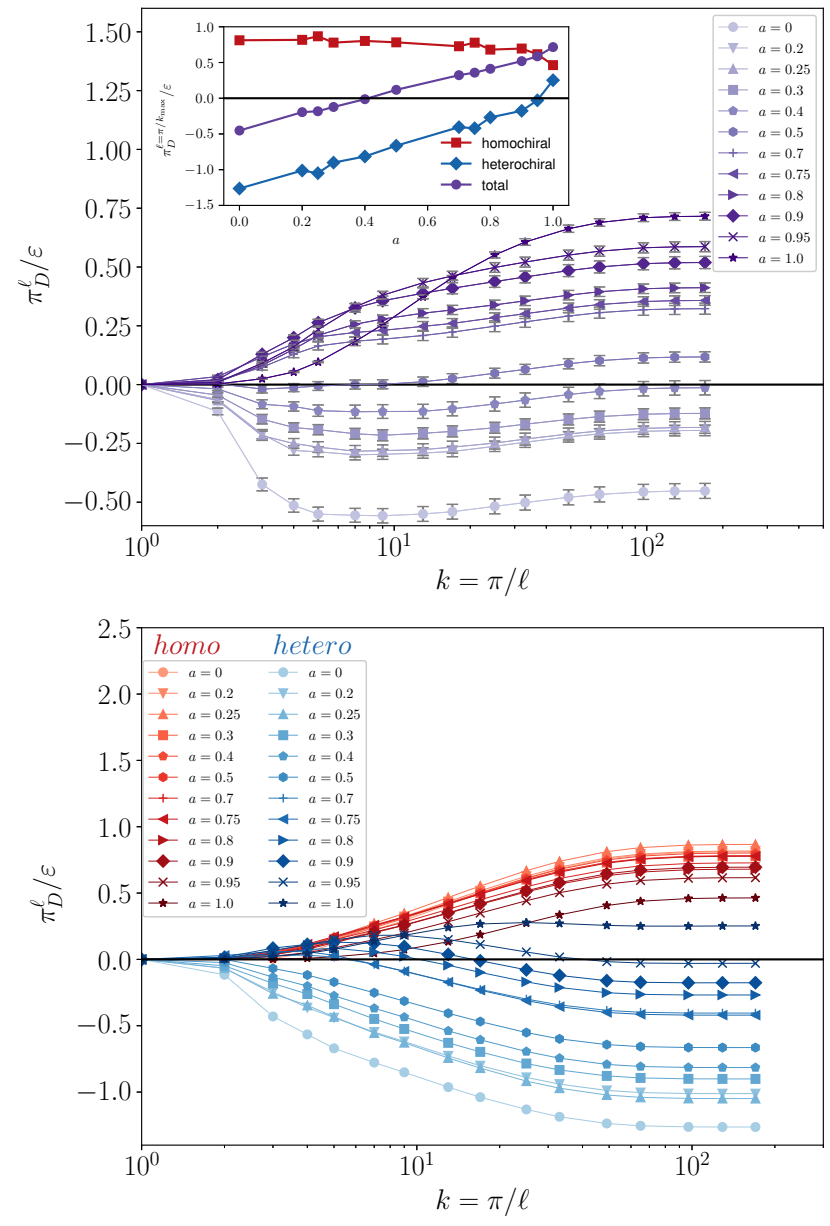

Figure 7. Top: $\pi_{D}^{\ell}$ versus the scale $k=\pi / \ell$. Bottom: decomposition of the curves shown in the top panel in heteroand homochiral contributions as a function of wavenumber. Inset top panel: total transfer $\pi_{D}^{\ell}$ with $\ell=\pi / k_{\max }$ and its decomposition in hetero- and homochiral contributions.

The bottom panel of the same figure shows that an even more universal behaviour is measured by looking at the flatness defined for the velocity field as:

$$
F_{v_{i}}=\frac{\left\langle\left(\partial_{i} v_{i}\right)^{4}\right\rangle}{\left\langle\left(\partial_{i} v_{i}\right)^{2}\right\rangle^{2}}=\frac{\left\langle S_{v_{i}}^{4}\right\rangle}{\left\langle S_{v_{i}}^{2}\right\rangle^{2}}
$$

and similarly for the magnetic field. Both curves have a very small dependency on $a$, supporting the conclusion that small-scale fluctuations in MHD are strongly universal, at least concerning the kind of forcing variations studied in this paper.

\section{CONCLUSIONS}

We have performed a systematic analysis of the total energy transfer in MHD at changing the large-scale forcing mechanisms, going from direct injection on the velocity field only to the case where stirring acts on the magnetic field only. We have split the total energy flux in 


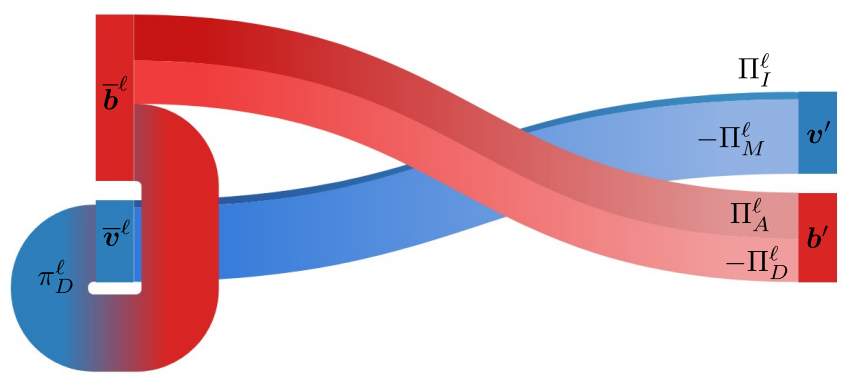

$a=0$

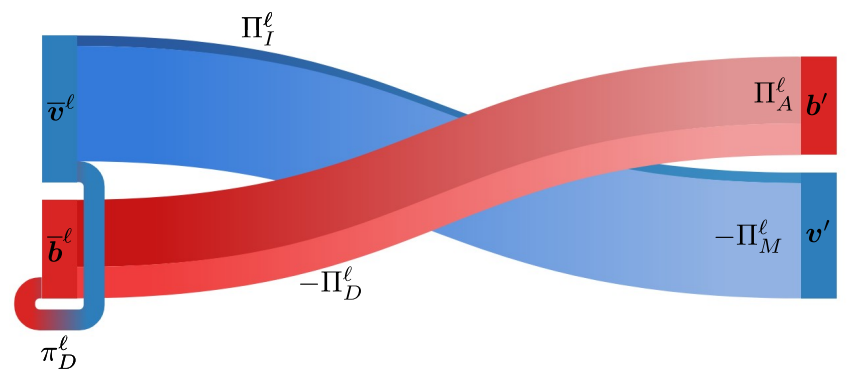

$a=0.5$

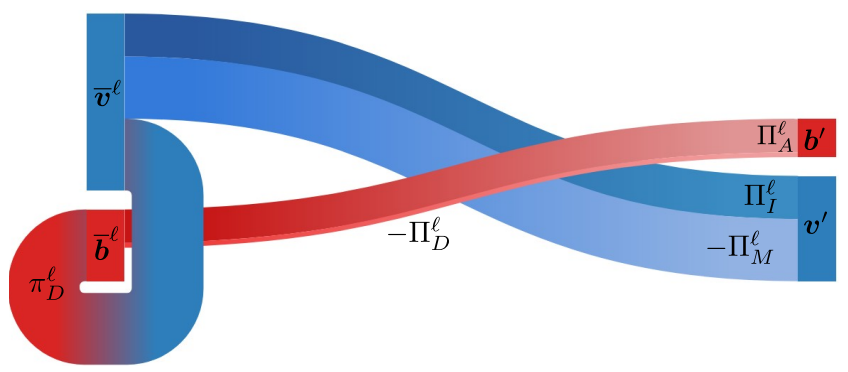

$a=1$

Figure 8. Maximal energy fluxes and large-scale conversion terms for $a=0, a=0.5$ and $a=1$. The line widths correspond to percentages of the total energy input $\varepsilon$. The direction of the transfers is indicated by the color gradient from dark to light. Dark blue: $\max _{\ell} \Pi_{I}^{\ell} / \varepsilon$, light blue: $\max _{\ell}-\Pi_{M}^{\ell} / \varepsilon$, dark red: $\max _{\ell} \Pi_{A}^{\ell} / \varepsilon$, light red: $\max _{\ell}-\Pi_{D}^{\ell} / \varepsilon$, gradient blue to red: $\max _{\ell}\left|\pi_{D}^{\ell}\right| / \varepsilon$. The sub-scale magnetic and velocity field fluctuations are denoted by $\boldsymbol{b}^{\prime}=\boldsymbol{b}-\overline{\boldsymbol{b}}^{\ell}$ and $\boldsymbol{v}^{\prime}=\boldsymbol{v}-\overline{\boldsymbol{v}}^{\ell}$, respectively. The diagrams have been created by adaptation of (Shanley 2019).

4 channels given by (i) the kinetic non-linear advection, (ii) the Lorentz force, (iii) the magnetic advection and (iv) magnetic stretching term and 2 sub-classes given by heterochiral and homochiral components for a total of 8 different sub-fluxes. We have shown that even a tiny injection of magnetic fluctuations at larger scales involves
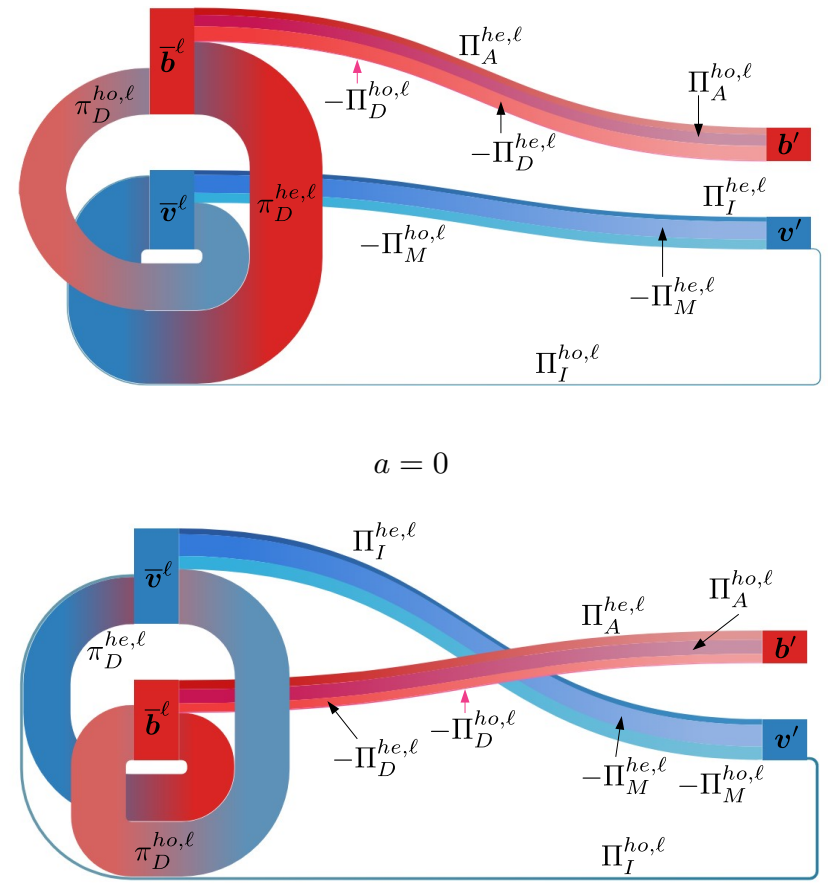

$a=0.5$

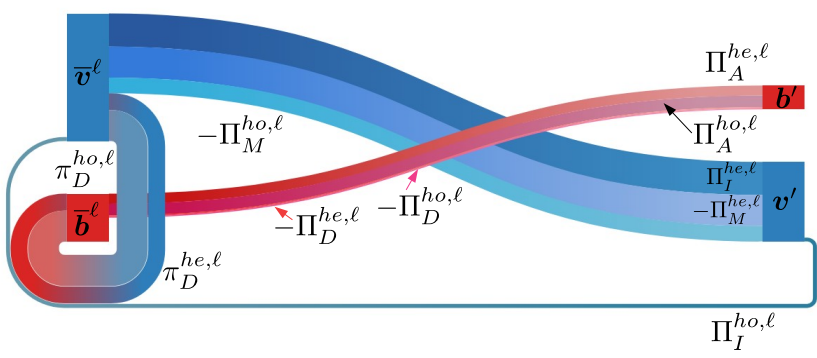

$a=1$

Figure 9. Helically decomposed maximal energy fluxes and large-scale conversion terms for $a=0, a=0.5$ and $a=1$. The line widths correspond to percentages of the total energy input $\varepsilon$. The direction of the transfers is indicated by the color gradient from dark to light. Dark blue: $\max _{\ell} \Pi_{I}^{h e, \ell} / \varepsilon$, dark cyan: $\max _{\ell} \Pi_{I}^{h o, \ell} / \varepsilon$, light blue: $\max _{\ell}-\Pi_{M}^{h e, \ell} / \varepsilon$, light cyan: $\max _{\ell}-\Pi_{M}^{h o, \ell} / \varepsilon$, dark red: $\max _{\ell} \Pi_{A}^{h e, \ell} / \varepsilon$, dark magenta: $\max _{\ell} \Pi_{A}^{h o, \ell} / \varepsilon$, light red: $\max _{\ell}-\Pi_{D}^{h e, \ell} / \varepsilon$, light magenta: $\max _{\ell}-\Pi_{D}^{h o, \ell} / \varepsilon$, gradient blue to red: $\max _{\ell}\left|\pi_{D}^{h e, \ell}\right| / \varepsilon$. gradient light blue to light red: $\max _{\ell}\left|\pi_{D}^{h o, \ell}\right| / \varepsilon$. The subscale magnetic and velocity field fluctuations are denoted by $\boldsymbol{b}^{\prime}=\boldsymbol{b}-\overline{\boldsymbol{b}}^{\ell}$ and $\boldsymbol{v}^{\prime}=\boldsymbol{v}-\overline{\boldsymbol{v}}^{\ell}$, respectively. The diagrams have been created by adaptation of (Shanley 2019). 

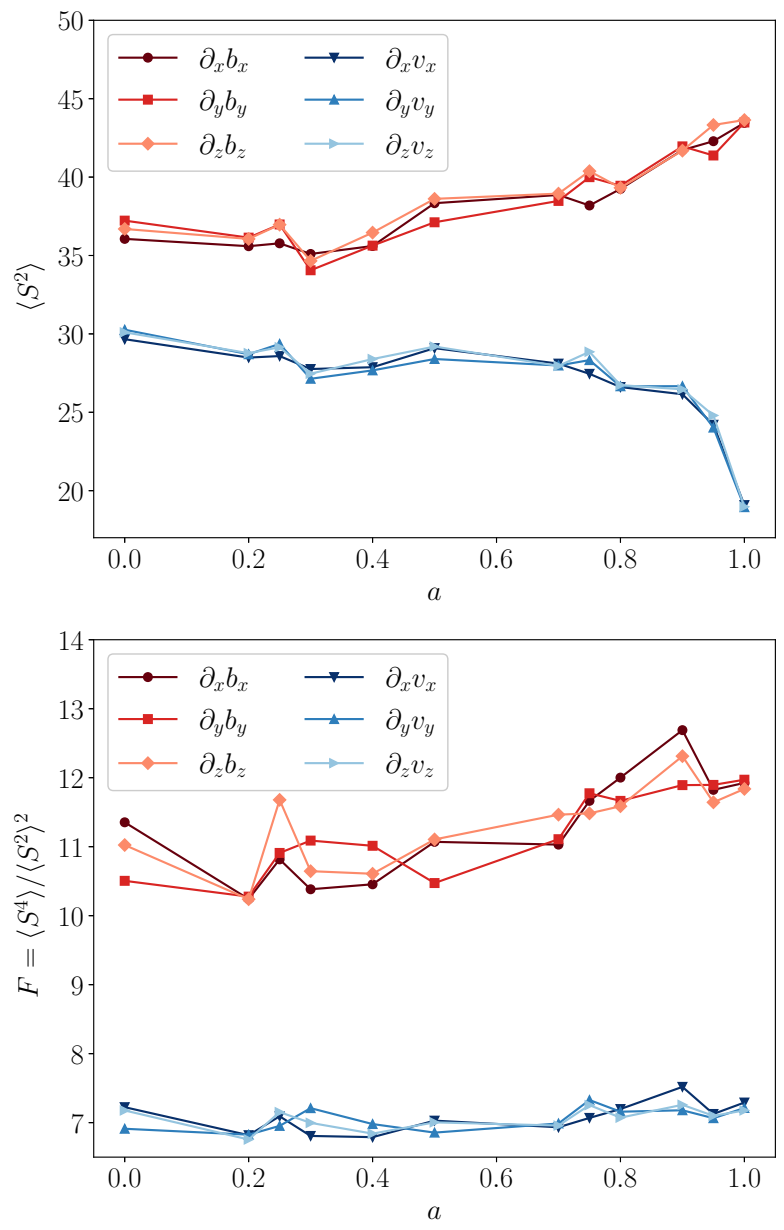

Figure 10. Top: second order moments of the three different longitudinal gradients for both velocity and magnetic field at changing $a$. Bottom: velocity and magnetic flatness for longitudinal gradients.

a quasi-singular response in the kinetic energy transfer mediated by the advection term, leading to an almost vanishing signal for the latter. We also show that this negligible mean flux is the result of a flux-loop balance between heterochiral (direct transfer) and homochiral (inverse transfer) channels. Conversely, both homochiral and heterochiral channels transfer energy forward for the other three channels. Furthermore, by increasing the relative amount of magnetic injection we observe a reduction of the energy transferred via the heterochiral interactions for all channels with the exception of the magnetic stretching channel. Cross exchange between velocity and magnetic field is reversed when the control forcing parameter is around. Small-scale properties are strongly universal, showing a non-trivial rearrangements of different transfer properties. Our decomposition approach is exact and can be extended to study the flux of other relevant quantities, such as magnetic and cross helicities and it can be useful for improving sub-gridmodelling and to understand the energy transfer mechanisms in the presence of different injection mechanisms.

\section{ACKNOWLEDGEMENTS}

This work has been supported by NSFC grant Nos. 91752201, 11672123, and 11902138; the Shenzhen Science and Technology Innovation Committee (grant No. KQTD20180411143441009) and the Department of Science and Technology of Guangdong Province (grant No. 2019B21203001). We acknowledge computing support provided by the Center for Computational Science and Engineering of Southern University of Science and Technology. Calculations for this research were partly conducted on the Lichtenberg High-performance computer of the TU Darmstadt, Germany. L.B. acknowledges the support from the Southern University of Science and Technology during his visit when we started the research presented in this work.

\section{APPENDIX A: HELICALLY DECOMPOSED FLUXES AND CONVERSION TERMS}

As can be seen from eqs. (26)-(27), the expansion of velocity and magnetic fields in their respective helical components involves projections onto subspaces spanned by $\boldsymbol{h}_{\boldsymbol{k}}^{ \pm}$, and these operations clearly commute with the filtering procedure defined in eq. (9). As such, eq. (24) can be directly substituted into the filtered MHD equations to obtain filtered evolution equations for the helical components. A subsequent projection onto one helical subspace yields

$$
\begin{aligned}
& -\partial_{j} \sum_{s_{2}, s_{3} \in\{+,-\}}\left(\overline{\overline{v_{i}^{s_{2}} \ell} \frac{\overline{v_{j}^{s_{3}}}}{\ell}}-\overline{\overline{b_{i}^{s_{2}} \ell}{\overline{b_{j}^{s}}}^{s_{3}} \ell}+\tau_{i j}^{\ell, I^{s_{2} s_{3}}}-\tau_{i j}^{\ell, M^{s_{2} s_{3}}}\right)^{s_{1}} \\
& +\nu \partial_{j j} \overline{v_{i}^{s_{1}} \ell}+\sqrt{a} \overline{f_{v_{i}}^{s_{1}} \ell}=\partial_{t} \overline{v_{i}^{s_{1}}} \ell,
\end{aligned}
$$

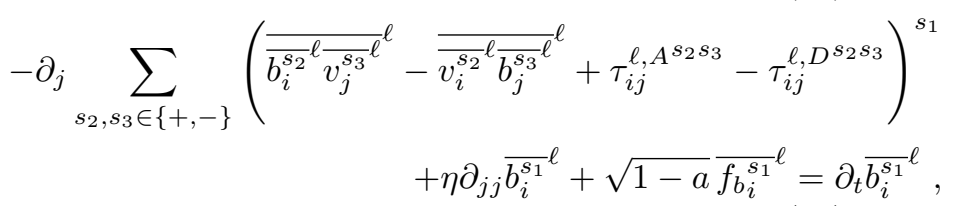

where $s_{1} \in\{+,-\}$ and we note the absence of a pressure term. Since the pressure gradient is orthogonal in the $L_{2}$-sense to the eigenfunctions $\boldsymbol{h}_{\boldsymbol{k}}^{ \pm}$, the projection onto a helical subspace renders the nonlinear term solenoidal and removes the pressure gradient. The helically decom- 
posed sub-filter-scale stress tensors read

$$
\begin{aligned}
& \tau_{i j}^{\ell, I^{s_{2} s_{3}}}=\overline{v_{i}^{s_{2}} v_{j}^{s_{3}}} \ell-\overline{\overline{v_{i}^{s_{2}} \ell \overline{v_{j}^{s_{3}}}} \ell}, \\
& \tau_{i j}^{\ell, M^{s_{2} s_{3}}}=\overline{b_{i}^{s_{2}} b_{j}^{s_{3}} \ell}-\overline{\overline{b_{i}^{s_{2}} \ell} \overline{b_{j}^{s_{3}}} \ell}{ }^{\ell}, \\
& \tau_{i j}^{\ell, A^{s_{2} s_{3}}}=\overline{b_{i}^{s_{2}} v_{j}^{s_{3}} \ell}-\overline{\overline{b_{i}^{s_{2}} \ell} \overline{v_{j}^{s_{3}}} \ell}, \\
& \tau_{i j}^{\ell, D^{s_{2} s_{3}}}=\overline{v_{i}^{s_{2}} b_{j}^{s_{3}} \ell}-\overline{\overline{v_{i}^{s_{2}} \ell} \overline{b_{j}^{s_{3}}} \ell},
\end{aligned}
$$

and following the same steps and in the derivation of the energy budget for the filtered components of velocity and magnetic field, one obtains four coupled energy budget equations for the filtered helical components

$$
\begin{aligned}
& 0=\frac{1}{2}\left\langle\frac{d}{d t}\left|\overline{v_{i}^{+}}\right|^{2}\right\rangle=-\left(\Pi_{I}^{+++}+\Pi_{I}^{++-}+\Pi_{I}^{+-+}+\Pi_{I}^{+--}\right) \\
& -\left(\pi_{I}^{+-+}+\pi_{I}^{+--}\right) \\
& +\left(\Pi_{M}^{+++}+\Pi_{M}^{++-}+\Pi_{M}^{+-+}+\Pi_{M}^{+--}\right) \\
& +\left(\pi_{M}^{+++}+\pi_{M}^{++-}+\pi_{M}^{+-+}+\pi_{M}^{+--}\right) \\
& +\sqrt{a}\left\langle\overline{v_{i}^{+}} \bar{f}_{v_{i}^{+}}^{\ell}\right\rangle \\
& 0=\frac{1}{2}\left\langle\frac{d}{d t}\left|\overline{v_{i}^{-}}\right|^{2}\right\rangle=-\left(\Pi_{I}^{---}+\Pi_{I}^{--+}+\Pi_{I}^{-+-}+\Pi_{I}^{-++}\right) \\
& -\left(\pi_{I}^{-+-}+\pi_{I}^{-++}\right) \\
& +\left(\Pi_{M}^{---}+\Pi_{M}^{--+}+\Pi_{M}^{-+-}+\Pi_{M}^{-++}\right) \\
& +\left(\pi_{M}^{---}+\pi_{M}^{--+}+\pi_{M}^{-+-}+\pi_{M}^{-++}\right) \\
& +\sqrt{a}\left\langle{\overline{v_{i}^{-}}}^{\ell} \overline{f_{v_{i}^{-}}^{-}} \ell\right\rangle \\
& 0=\frac{1}{2}\left\langle\frac{d}{d t}\left|\overline{b_{i}^{+}}\right|^{2}\right\rangle=-\left(\Pi_{A}^{+++}+\Pi_{A}^{++-}+\Pi_{A}^{+-+}+\Pi_{A}^{+--}\right) \\
& -\left(\pi_{A}^{+-+}+\pi_{A}^{+--}\right) \\
& +\left(\Pi_{D}^{+++}+\Pi_{D}^{++-}+\Pi_{D}^{+-+}+\Pi_{D}^{+--}\right) \\
& +\left(\pi_{D}^{+++}+\pi_{D}^{++-}+\pi_{D}^{+-+}+\pi_{D}^{+--}\right) \\
& +\sqrt{1-a}\left\langle\overline{b_{i}^{+}} \ell \overline{f_{b_{i}^{+}}} \ell\right\rangle \\
& 0=\frac{1}{2}\left\langle\frac{d}{d t}\left|\overline{b_{i}^{-}}\right|^{2}\right\rangle=-\left(\Pi_{A}^{---}+\Pi_{A}^{--+}+\Pi_{A}^{-+-}+\Pi_{A}^{-++}\right) \\
& -\left(\pi_{A}^{-+-}+\pi_{A}^{-+}\right) \\
& +\left(\Pi_{D}^{---}+\Pi_{D}^{--+}+\Pi_{D}^{-+-}+\Pi_{D}^{-++}\right) \\
& +\left(\pi_{D}^{---}+\pi_{D}^{--+}+\pi_{D}^{-+-}+\pi_{D}^{-++}\right) \\
& +\sqrt{1-a}\left\langle\overline{b_{i}^{-}} \ell \overline{f_{b_{i}}^{-}} \ell\right\rangle
\end{aligned}
$$

where the superscript $\ell$ has been dropped to simplify the notation of the fluxes and conversion terms. The helically decomposed fluxes and resolved-scale conversion terms are defined as

$$
\begin{aligned}
& \Pi_{I}^{s_{1} s_{2} s_{3}}=-\left\langle\left(\partial_{j} \overline{v_{i}^{s_{1}}}\right) \tau_{i j}^{\ell, I^{s_{2} s_{3}}}\right\rangle, \\
& \Pi_{M}^{s_{1} s_{2} s_{3}}=-\left\langle\left(\partial_{j} \overline{v_{i}^{s_{1}} \ell}\right) \tau_{i j}^{\ell, M^{s_{2} s_{3}}}\right\rangle, \\
& \Pi_{A}^{s_{1} s_{2} s_{3}}=-\left\langle\left(\partial_{j} \overline{b_{i}^{s_{1}} \ell}\right) \tau_{i j}^{\ell, A^{s_{2} s_{3}}}\right\rangle, \\
& \Pi_{D}^{s_{1} s_{2} s_{3}}=-\left\langle\left(\partial_{j} \overline{b_{i}^{s_{1}} \ell}\right) \tau_{i j}^{\ell, D^{s_{2} s_{3}}}\right\rangle,
\end{aligned}
$$

and

$$
\begin{aligned}
& \pi_{I}^{s_{1} s_{2} s_{3}}=-\left\langle\left(\partial_{j} \overline{v_{i}^{s_{1}} \ell}\right) \overline{v_{i}^{s_{2}} \ell} \overline{v_{j}^{s_{3}}} \ell\right\rangle \\
& =\left\langle\left(\partial_{j} \overline{v_{i}^{s_{2}} \ell}\right) \overline{v_{i}^{s_{1}} \ell} \overline{v_{j}^{s_{3}} \ell}\right\rangle \\
& =-\pi_{I}^{s_{2} s_{1} s_{3}}, \\
& \pi_{A}^{s_{1} s_{2} s_{3}}=-\left\langle\left(\partial_{j} \overline{b_{i}^{s_{1}} \ell}\right) \overline{b_{i}^{s_{2}} \ell} \overline{v_{j}^{s_{3}} \ell}\right\rangle \\
& =\left\langle\left(\partial_{j} \overline{b_{i}^{s_{2}} \ell}\right) \overline{b_{i}^{s_{1}} \ell} \overline{v_{j}^{s_{3}} \ell}\right\rangle \\
& =-\pi_{A}^{s_{2} s_{1} s_{3}} \text {, } \\
& \pi_{D}^{s_{1} s_{2} s_{3}}=-\left\langle\left(\partial_{j} \overline{b_{i}^{s_{1}} \ell}\right) \overline{v_{i}^{s_{2}} \ell} \overline{b_{j}^{s_{3}} \ell}\right\rangle \\
& =\left\langle\left(\partial_{j} \overline{v_{i}^{s_{2}} \ell}\right) \overline{b_{i}^{s_{1}} \ell} \overline{b_{j}^{s_{3}} \ell}\right\rangle \\
& =-\pi_{M}^{s_{2} s_{1} s_{3}} \text {, }
\end{aligned}
$$

A few observations can be made from these expressions and subsequently from eqs. (39) - (42). First, eqs. (47) and (48) imply that $\pi_{I}^{ \pm \pm \pm}, \pi_{I}^{ \pm \pm \mp}, \pi_{A}^{ \pm \pm \pm}$, and $\pi_{A}^{ \pm \pm \mp}$ vanish, as these terms can be written as total gradients. Second, the remaining terms $\pi_{I}^{ \pm \mp \pm}=-\pi_{I}^{\mp \pm \pm}$ exchange kinetic energy between $\overline{\boldsymbol{v}^{+}}$and $\overline{\boldsymbol{v}^{-}}$, as can be seen from eqs. (39) and (40), which is the only kinetic energy exchange between positively and negatively helical sectors. The helically decomposed energy fluxes defined in eqs. (43) - (46) conserve kinetic and magnetic energy for each helical component separately, which are categorized into homochiral or heterochiral fluxes, depending whether the three fields entering in the expressions (43) - (46) have all the same chirality or there is one with opposite chirality of the other two. It is trivial to obtain the energy balance equation for the filtered velocity and magnetic field by summing up eqs. (39) - (42),

$$
\begin{aligned}
0= & -\left(\Pi_{I}^{h o, \ell}+\Pi_{I}^{h e, \ell}\right)+\left(\Pi_{M}^{h o, \ell}+\Pi_{M}^{h e, \ell}\right)+\left(\pi_{M}^{h o, \ell}+\pi_{M}^{h e, \ell}\right) \\
& +\sqrt{a}\left\langle\bar{v}_{i}^{\ell}{\overline{f_{v i}}}^{\ell}\right\rangle-\varepsilon_{v}^{\ell}, \\
0= & -\left(\Pi_{A}^{h o, \ell}+\Pi_{A}^{h e, \ell}\right)+\left(\Pi_{D}^{h o, \ell}+\Pi_{D}^{h e, \ell}\right)+\left(\pi_{D}^{h o, \ell}+\pi_{D}^{h e, \ell}\right) \\
& +\sqrt{1-a}\left\langle\bar{b}_{i}^{\ell} \overline{f_{b_{i}}}\right\rangle-\varepsilon_{b}^{\ell} .
\end{aligned}
$$




\section{REFERENCES}

Alexakis, A. 2013, Phys. Rev. Lett., 110, 084502

Alexakis, A. 2017, J. Fluid. Mech., 812, 752

Alexakis, A., \& Biferale, L. 2018, Physics Reports, 767, 1

Aluie, H. 2017, New J. Physics, 19, 025008

Aluie, H., \& Eyink, G. L. 2009a, Phys. Fluids, 21, 115107

Aluie, H., \& Eyink, G. L. 2009b, Phys. Fluids, 21, 115108

Barnes, A. 1979, in Solar System Plasma Physics, vol. I, ed. E. N. Parker, C. F. Kennel, \& L. J. Lanzerotti

(Amsterdam: North-Holland), 251

Beresnyak, A., \& Lazarian, A. 2009, Astrophys. Journal, 702, 1190

Bian, X., \& Aluie, H. 2019, Physical review letters, 122, 135101

Biferale, L., Musacchio, S., \& Toschi, F. 2012, Phys. Rev. Lett., 108, 164501

Biskamp, D. 2003, Magnetohydrodynamic Turbulence., 1st edn. (Cambridge University Press)

Boldyrev, S. 2005a, ApJ, 626, L37

Boldyrev, S. 2005b, Phys. Rev. Lett., 96, 115002

Boldyrev, S., Mason, J., \& Cattaneo, F. 2009, ApJ, 699, L39

Brandenburg, A. 2001, Astrophys. J., 550, 824

Brandenburg, A., \& Subramanian, K. 2005, Physics Reports, 417, 1

Bray, R., Loughhead, R., \& Durrant, C. 1984, The Solar Granulation, Cambridge Astrophysics (Cambridge University Press).

https://books.google.com/books?id=ZKGjQgAACAAJ

Bruno, R., \& Carbone, V. 2005, Living Reviews in Solar Physics, 2, 4

Bruno, R., \& Carbone, V. 2013, Living Reviews in Solar Physics, 10, 2

Buzzicotti, M., Linkmann, M., Aluie, H., et al. 2018, J. Turbul., 19, 167

Chen, C. 2016, Journal of Plasma Physics, 82

Chernyshov, A. A., Karelsky, K. V., \& Petrosyan, A. S. 2010, Phys. Plasmas, 17, 102307

Chertkov, M., Falkovich, G., Kolokolov, I., \& Vergassola, M. 1999, Phys. Rev. Lett., 83, 4065

De Pietro, M., Biferale, L., \& Mailybaev, A. A. 2015, Phys. Rev. E, 92, 043021

Dikpati, M., \& Gilman, P. A. 2001, The Astrophysical Journal, 552, 348

Galtier, S. 2016, Introduction to modern magnetohydrodynamics (Cambridge University Press)

Galtier, S., Nazarenko, S. V., Newell, A. C., \& Pouquet, A. 2000, J. Plasma Phys., 63, 447

Galtier, S., Nazarenko, S. V., Newell, A. C., \& Pouquet, A. 2002, Astrophys. J., 564, L49
Gilman, P. A. 2000, The Astrophysical Journal Letters, 544, L79

Goldreich, P., \& Sridhar, S. 1995, Astrophys. J., 438, 763

Goldstein, M. L., Roberts, D. A., \& Matthaeus, W. 1995, Annual review of astronomy and astrophysics, 33, 283

Grappin, R., \& Müller, W.-C. 2010, Phys. Rev. E, 82, 026406

Grete, P., O’Shea, B. W., \& Beckwith, K. 2020, arxiv:2009.03342

Horbury, T., Forman, M., \& Oughton, S. 2005, Plasma physics and controlled fusion, 47, B703

Iroshnikov, P. S. 1964, Soviet Astronomy, 7, 566

Kessar, M., Balarac, G., \& Plunian, F. 2016, Phys. Plasmas, 23, 102305

Klein, K. G., Alexandrova, O., Bookbinder, J., et al. 2019, [Plasma 2020 Decadal] Multipoint Measurements of the Solar Wind: A Proposed Advance for Studying Magnetized Turbulence. https://arxiv.org/abs/1903.05740

Kraichnan, R. H. 1965, Phys. Fluids, 8, 1385

Kraichnan, R. H. 1994, Physical review letters, 72, 1016

Leighton, R. B. 1963, Annual review of astronomy and astrophysics, 1, 19

Leighton, R. B., Noyes, R. W., \& Simon, G. W. 1962, The Astrophysical Journal, 135, 474

Lessinnes, T., Plunian, F., \& Carati, D. 2009, Theor. Comput. Fluid Dyn., 23, 439

Linkmann, M., Sahoo, G., McKay, M., Berera, A., \& Biferale, L. 2017, Astrophys. J., 836, 26

Linkmann, M. F., Berera, A., McKay, M. E., \& Jäger, J. 2016, J. Fluid Mech., 791, 61

Mason, J., Cattaneo, F., \& Boldyrev, S. 2006, Phys. Rev. Lett., 97, 255002

Matthaeus, W. H., \& Zhou, Y. 1989, Phys. Fluids B, 1, 1929

Matthaeus, W. H., Bandyopadhyay, R., Brown, M. R., et al. 2019, [Plasma 2020 Decadal] The essential role of multi-point measurements in turbulence investigations: the solar wind beyond single scale and beyond the Taylor Hypothesis. https://arxiv.org/abs/1903.06890

McKay, M. E., Linkmann, M., Clark, D., Chalupa, A. A., \& Berera, A. 2017, Phys. Rev. Fluids, 2, 114604

Miesch, M., Matthaeus, W., Brandenburg, A., et al. 2015, Space Sci. Rev., 194, 97

Miesch, M. S. 2005, Living Reviews in Solar Physics, 2, 1

Mininni, P. D. 2011, Annu. Rev. Fluid Mech., 43, 377

Moffatt, H. K. 1978, Magnetic Field Generation in Electrically Conducting Fluids (Cambridge University Press) 
Müller, W.-C., \& Biskamp, D. 2000, Phys. Rev. Lett., 84, 03475

Müller, W.-C., \& Carati, D. 2002, Comput. Phys. Commun., 147, 544

Offermans, G. P., Biferale, L., Buzzicotti, M., \& Linkmann, M. 2018, Phys. Plasmas, 25, 122307

Parker, E. N. 1979, Cosmical Magnetic Fields: their Origin and their Activity (Oxford, Clarendon Press; New York, Oxford University Press)

Rathmann, N. M., \& Ditlevsen, P. D. 2017, Phys. Rev. Fluids, 2, 054607

Rathmann, N. M., \& Ditlevsen, P. D. 2019, Astrophys. J., 887, 95

Rieutord, M., \& Rincon, F. 2010, Living Reviews in Solar Physics, 7, 2

Shanley, T. 2019, d3-sankey-circular, https://github.com/tomshanley/d3-sankey-circular, GitHub
Steenbeck, M., Krause, F., \& Rädler, K.-H. 1966, Z. Naturforsch. A, 21, 369

Teissier, J.-M., \& Müller, W.-C. 2020, arxiv:2009.09374

Tobias, S. M., Cattaneo, F., \& Boldyrev, S. 2013, in Ten Chapters in Turbulence (Cambridge University Press), 351-404

Tu, C.-Y., \& Marsch, E. 1995, Space Science Reviews, 73, 1 Vergassola, M. 1996, Physical Review E, 53, R3021

Vreman, B., Geurts, B., \& Kuerten, H. 1994, J. Fluid Mech., 278, 351

Waleffe, F. 1992, Phys. Fluids A, 4, 350

Waleffe, F. 1993, Phys. Fluids A, 5, 677

Yang, Y., Shi, Y., Wan, M., Matthaeus, W. H., \& Chen, S. 2016, Phys. Rev. E, 93, 061102

Zhou, Y., Matthaeus, W., \& Dmitruk, P. 2004, Reviews of Modern Physics, 76, 1015

Zhou, Y., \& Vahala, G. 1991, J. Plasma Phys., 45, 239 